\title{
Nursing interventions in the extubation process: a scoping review
}

\begin{abstract}
Background: One of the main reasons why people need to be in an intensive care unit is due to the need of invasive mechanical ventilation. Mechanical ventilation allows for assisting or replacing spontaneous breathing. Weaning is a critical phase of mechanical ventilation and it represents about $40 \%$ of it. Ventilatory extubation is essential, not only because it is an indicator of recovery of the person's ventilatory autonomy, but also because of the risks that prolonged invasive mechanical ventilation causes.
\end{abstract}

Objectives: This research report was made due to the need of scientific evidence for the extubation process. We aim to get further insight into the nursing cares in people submitted to invasive mechanical ventilation, in intensive care units, in order to optimize this process.

Design: It was made a scoping review, based on the recommended methodology of Joanna Briggs Institute. It was used a five-step process that included: Identifying the research question; Identifying relevant studies; Study selection; Charting the data and collating, Summarizing and reporting the results.

Data sources: A literature search was performed in the following databases: Cochrane, CINAHL, Nursing Reference Center,Medline, ISI, Scielo and RCAAP.

Methods: Two independent reviewers assessed eligibility based on the article's titles, abstracts and full text. Data extraction was conducted based on a table developed by the reviewers

Results: Nine studies were included in this review. Circadian cycle, nurse-patient relationship, criteria, sedation, spontaneous breathing trial, secretions, level of consciousness, anxiety, vital signs, risk factors, oxygenation, general assessment of the patient, stridor, oral hygiene, position, extubation and food interruption were the relevant topics that emerged from each article.

Conclusion: It is shown that the vision and the holistic intervention during the extubation process is essential for the improvement of the quality of the ventilatory weaning and consequent extubation. This study allows for gathering the best existing scientific evidence to provide nursing cares of quality and excellence, minimizing the associated risks, by informing the reader in a schematic form, of the nursing care in the various stages of the extubation process.

Keywords: airway extubation, intensive care units, nurses, respiration, artificial
Volume 5 Issue 6 - 2020

\author{
Maria Beatriz Rocha,' Ana Filipa Poeira, ${ }^{2}$ \\ Raquel Flamino,' Neuza Santos' \\ 'Department of Nursung, School of Health, Polytechnic Institute \\ of Setúbal, Portugal \\ ${ }^{2}$ Department of Nursung, School of Health, Polytechnic Institute \\ of Setúbal, Researcher in NURSE' IN Nursing Research Unit for \\ South and Islands, Portugal
}

Correspondence: Maria Beatriz Rocha, Department of Nursing, School of Health, Polytechnic Institute of Setúbal, Portugal, Tel 00351934069I7I,

Email mariabeatriz.Ir@hotmail.com

Received: October 22, 2020 | Published: December 15, 2020

\section{What is already known about the topic?}

The risks associated with invasive mechanical ventilation. Extubation is a critical phase and it's failure requires endotracheal re-intubation,whichincreases hospital mortality and length of stay in the intensive care unit.

\section{What this paper adds?}

The complexity of the Invasive Mechanical Ventilation and the extubation process requires from nurses a differentiated intervention that responds to the needs of the person/family.

Compilation and layout of nursing care in the various stages of the extubation process: pre-extubation; extubation; post extubation.

\section{Introduction}

One of the main reasons of hospital stay in an intensive careunit, is the need to invasive mechanical ventilation, which is a process that allows to assist or replace spontaneous breathing. ${ }^{1}$ It aims to support gas exchange, to reduce respiratory muscle fatigue, to diminish oxygen consumption, to reduce respiratory distress and to apply specific therapies. ${ }^{2}$

An epidemiological study conducted in a range ofintensive care units, revealed that 217 out of 390 hospitalized patientswere submitted to invasive mechanical ventilation. ${ }^{3}$

The need of invasive mechanical ventilation requires complex and diversified nursing cares. Nurses have the responsibility of providing a continuous care in order to support a positive evolution. (Fogaça \& Silva, 2010)

There are several risks associated to invasive mechanical ventilation, such as bronchoaspiration, barotrauma, ventilatorassociated pneumonia, decreased cardiac output, and respiratory muscle dysfunction. ${ }^{4}$

Ventilatory extubation is essential, not only because it is an indicator of recovery of the person's ventilatory autonomy, but also because of the risks that prolonged invasive mechanical ventilation causes. (Simões, 2016)At 48 hours, it's failure rates vary between 15 and 18 per cent,requiring endotracheal re-intubation, which increases hospital mortality and length of stay in the intensive care unit. ${ }^{6}$ 
An exploratory study showed that about 15 per cent of intubated patients needed re-intubation caused by extubation failure. This implied 12 more days on invasive mechanical ventilation. The same study concluded that the mortality rate was higher in re-intubated patients $(43 \%)$, compared to successful extubated patients $(12 \%){ }^{7}$

The person submitted to invasive mechanical ventilation requires thorough nursing care. Nonetheless, nurses mentioned the lack of knowledge and safety, insufficient time to learn and lack of opportunities.

This scoping review was made based on the methodology presented at the Joanna Briggs Institute for scoping reviews, in order to identify nursing cares in the process of ventilatory extubation. More specifically, this review aims to answer the following questions:

- What are the nursing interventions for the person in an intensive care unit in pre-extubation?

- What are the nursing interventions for the person in an intensive care unit during extubation?

- What are the nursing interventions for the person in an intensive care unit after extubation?

It should also be noted that the objectives, inclusion criteria and method of analysis of this scoping review were previously specified and documented in a research report.

\section{Methods}

Evidence-based health care is an approach reflected in the search for better and reasoned evidence, in order to act correctly, effectively and to the highest possible standards. ${ }^{9}$ A scoping reviewallow us to have a bigger broader range of literature analysis because it aims to identify both published and unpublished (grey literature) primary studies as well as reviews, providing a more complete understanding of the topic of interest, as its objectives focus on mapping existing evidence on a particular topic to inform decision making and to improve clinical care. ${ }^{10}$

The scoping review questions were based upon the "PCC" mnemonic, which stands for the Population, Concept and Context. Population of all studies refers to adult people, from both genders, orotracheally intubated. The concept means nursing interventions for the optimization of the extubation process. The context refers to intensive care units, without any cultural or geographical specification.

\section{Search strategy}

A three-step search strategy was utilized. The first step is an initial limited search of databases relevant to the topic, followed by an analysis of the text words contained in the title and abstract of retrieved papers and of the index terms used to describe the articles. The second step is a second search in all included databases: Cochrane, CINAHL, Nursing Reference Center, RCAAP, Medline, ISI and Scielo, using the indexed vocabulary, if any, as a sensitive search strategy. (Table 1) Thirdly, it was searched the reference list of all included articles for additional studies. We contacted an author of a primary study, who allowed us to have complete access to his article.

Only studies published in English and Portuguese were considerate for inclusion. The timeframe used for the search was between the year of 2015 to 2019. Studies that included rehabilitation nursing cares, people submitted to tracheotomy and children were excluded.

Results of each article were compared and analyzed by two independent reviewers. Disagreements were argued with a third reviewer.

The Joanna's Briggs Institute PRISMA Flow Diagram for the scoping review process is presented below in Figure 1. As presented, the research identified 79 relevant studies to be included. Of these, 69 studies were excluded after reading and analyzing the title and abstract. Of the remaining articles, one of them was excluded after reading the full text, because it didn't meet the inclusion criteria. We included 9 studies in the review.

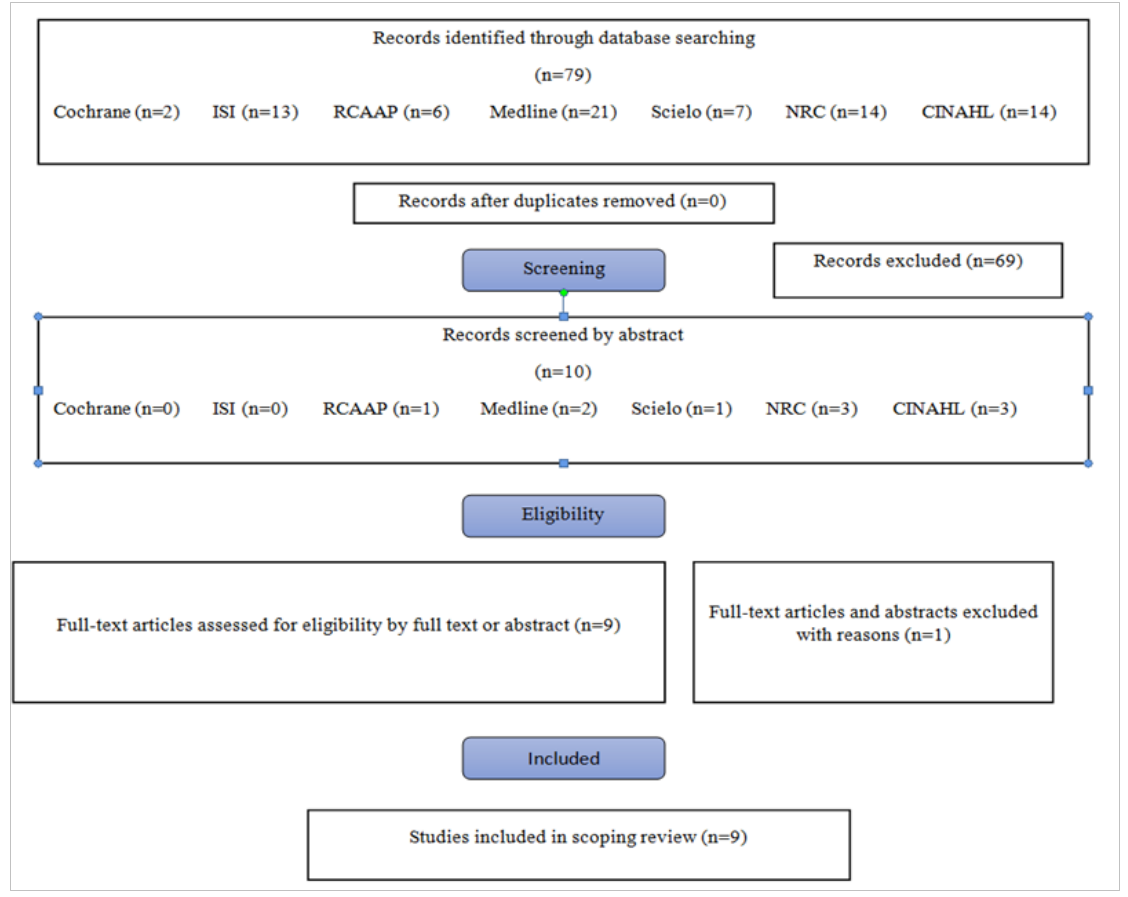

Figure I PRISMA flow diagram for the scoping review process. 


\section{Data extraction}

Extraction data from all articles was made by at least two independent reviewers, based on the objective and sub-questions ofthis scoping review. All disagreements werere solved through discussion or with a third reviewer.

The following table presents the identification of each study using the PCC method, the level of evidence, the time period of each study and their division according to the 3 stages of the extubation process, with the Nursing interventions addressed by each study in the different stages.

A scoping review is not intended to assess the methodological quality of included studies, even though the level of evidence for each of the included studies has been presented in data extraction (Table 2).

\section{Results}

In order to answer the research question, it is shown the main results of the analysis of allstudies, sorted by theme and chronologically.

\section{Pre-extubation}

\section{Nurse-patient relationship}

According to the empirical literature, the therapeutic relationship promoted within the scope of nursing professional practice is characterized by the partnership established with the person. Itaims for help them to be proactive in achieving their health plan, through a dynamic process. It is nurse's responsibility for providing quality continuous care so that there is a positive evolution of the person. ${ }^{11-14}$

\section{Criteria}

Regarding the use of a list of extubation criteria, the person must meet specific criteria for ventilatory weaning. In the articles analyzed, one of them states that a list of criteria cannot be applied due to the constant and complexity changes in the person's health status. ${ }^{11}$ On the other hand, the remaining articles state that there is a need for weaning criteria, improving its quality. ${ }^{12-17}$

\section{Risk factors}

The framework presented refers the need to require from nurses a clinical act for an early detection of potentially dangerous situations. Identifying the risk factors for prolonged weaning, such as hypercapnia, muscle weakness, tachycardia and the reduction in the Glasgow Coma Scale, allows an adequate performance and greeter treatment effectiveness. ${ }^{12,17}$

\section{Sedation}

Some of the articles say that nurses must evaluate the level of sedation of the person submitted to invasive mechanical ventilation, because it is essential. ${ }^{11,13} \mathrm{~A}$ high level of sedation is considered a barrier in the extubation process. ${ }^{11}$

\section{Spontaneous breathing trial}

The framework presented states that it is essential to verify the adaption of the person to spontaneous breathing, in order to reduce the rate of extubation failure and prevent re-intubation. The Spontaneous Breathing Trial allows us to verify this adaptation. Both articles analyzed address the use of the $\mathrm{T}$ piece in Spontaneous Breathing Trial. According to the literature, it is a primordial trial that is used before extubation in order to optimize its success. ${ }^{12,14}$

\section{Food interruption}

Based in the analysis of the articles, it is necessary that the person is in food interruption for at least 4 hours before extubation, in order to prevent complications. ${ }^{16}$

\section{Secretions}

The framework presented addresses the need of thorough nursing cares for the person submitted to invasive mechanical ventilation and the importance of the nursing interventions to identify and manage the most common problems in the critically ill patient. Prior to the extubation, the nurses must evaluate the presence of excessive secretions and aspirate them. ${ }^{15,16}$

\section{Stridor}

Rapid problem recognition and quality nursing cares can resolve acute respiratory distress, dyspnea, increased breathing effort, and prevent adverse events. Only one article deals with nursing interventions that prevent the occurrence of laryngeal edema and consequent stridor, having obtained positive results in decreasing stridor rate after extubation, re-intubation rate and re-intubation rate due to stridor after extubation. ${ }^{17}$

\section{Circadian cycle}

With the data collected and the benefits presented in the various studies it is possible to state that the best period of the day for extubation will be the morning. ${ }^{13,15,17}$ Although, one of the studies refers that the interruption of ventilatory weaning during the night may delay the person's evolution. ${ }^{11}$

\section{Extubation}

\section{Level of conscience}

The framework presented addresses the need of evaluate the level of conscience of the person submitted to orotracheal intubation. During the extubation, the nurse should evaluate the level of conscience in order to always ensure a score greater than 13 points on the Glasgow Coma Scale and ensuring minimum sedation as possible. ${ }^{15}$

\section{Anxiety}

Nurse's should act in order to reduce the person's anxiety by combining a signal to indicate the need to stop the extubation, teaching stress reduction techniques and ensuring that the family is present if possible. ${ }^{16}$

\section{Secretions}

The framework presented addresses the need of rigorous nursing cares to the people submitted to orotracheal intubation and the importance to identify and to manage the most common problems of mechanical invasive ventilation. Two articles state that during extubation, nurses must firstly confirm the presence of cough reflex and the swallowing ability. After that, the patient must be instructed to cough at extubation and to cough again after the orotracheal tube is removed, in order to expel the secretions..$^{15,16}$

\section{Positioning}

During the extubation, the person should be positioned supine, with the elevation of the headboard. ${ }^{16}$

\section{Stridor}

Some articles refer that the prevention of laryngeal edema and consequent stridor is possible by administering, according to medical 
prescription, $1 \mathrm{mg}$ of budesonide by nebulization immediately prior to extubation. ${ }^{17}$

\section{Extubation}

It is state that nurses should remove the tape that secure the orotracheal tube and then deflate the cuff. ${ }^{16}$

\section{Vital signs}

According to the empirical literature, nursing caresallows to early predict and detect complications, in order to ensure the evolution of the person and accurate efficient and timely interventions to promote people safety. During the extubation process, the nurses must monitor vital signs such as pain, though the pain assessment scale, level of oxygen saturation and the physiological state in order to detect risk situations. ${ }^{15}$

\section{Post-extubation}

\section{Positioning}

The person should be positioned in fowler, in order to promote adequate oxygenation. ${ }^{15,16}$

\section{Oxygenation}

According to the articles, the person should be immediately connected to an oxygen mask after removing the tube.Nurses should encourage the person to inhale and exhale deeply for a more effective ventilation. Post-extubation oxygen saturation levels should be checked, as well as the person's response to the extubation. ${ }^{15,16}$

\section{General assessment of the patient}

According to the frame of reference, there should be a continuous assessment after extubation, because the patient may have changes in their clinical and respiratory status. According to the articles, in the post-extubation period, it is essential to do an analysis of the general state of the person, including vital signs and respiratory distress. ${ }^{15,16}$

\section{Oral hygiene}

In addition to the need of a protocol regarding to the weaning, a regular oral hygiene protocol reduces the risk of oral colonization, which may lead to further complications.

Brushing each oral quadrant and a tongue wash with a proper brush should be done in at least two minutes. These procedures should be performed twice times a day, as well as flossing and applying moisturizing lipstick on the lips. The person should also be instructed to rinse with an oral elixir, if possible..$^{18,19}$

\section{Discussion}

\section{Pre-extubation interventions discussion}

The pre-extubation interventions that the analysis of the studies presented, allow us to conclude that a continuous care during the extubation process, is the patient's basic needs during this process. A continuous and effective communication, a physical presence, a face to face contact, psychological support and a holistic perspective are pointed out as an important need to patient's readiness to weaning. Also, an effective communication between the shifts and the experience of nurses are factors that may increase the chances of a successful extubation.

A use of a validated and reliable tool or list of criteria reduces the risk of premature reduction of ventilatory support, decreases the duration of mechanical ventilation, the time before first weaning attempt, the length of hospital stay and the number of patients that requires ventilation for more than 21 days. The patient's readiness to weaning can also be determine by the evaluation of risk factors, for example measures of muscle weakness, such as handgrip strength and oxidative stress that are associated with difficult or prolonged weaning. Also, another point that can complicate weaning is an excessive sedation.

Once weaning readiness criteria have been met, a spontaneous breathing trial must be conduct for a minimum of 30 minutes and 2 hours maximum. It allows for predict a patient's tolerance of extubation, and it involves reduction of ventilatory support to a minimum level using a T-piece. A T-piece allows for supplying humidified oxygen for a predetermined period.

Nurses must verify that feeding tube infusions have been stopped at least 4 hours prior to the planned time of extubation, in order to prevent complications, such as aspiration pneumonia and aspiration of vomit.

Nurses must also promote the mobilization and remove excessive secretions, if necessary. They also must monitor signs of respiratory distress and vital signs, in order to determine the person's readiness to extubation. Patient's must demonstrate an adequate oxygenation, and stable hemodynamic and metabolic status.

Weaning is a daily practice that occurs particularly in the morning, according to circadian cycle. During the night shift, there are some strategies that nurses may adopt, in order to improve the weaning process, such as close monitoring of nurse's previous reports, nurse support and controlled sedation.

\section{During extubation interventions discussion:}

Nurses must confirm the level of conscience of the patients, and guarantee they are arousable, with a Glasgow Coma Scale greater than 13 points. They also must confirm the presence of a cough reflex and the ability to swallow, which indicates the patient can protect his airway.

Regarding to extubation, the patient can present anxiety and nurses must assess the patient's understanding of extubation and explain how it is going to be made, all the steps of the procedure, answer any questions and provide emotional support. Stress reduction techniques can also be taught, such as combining a signal to indicate the need to stop the process, if needed.

The patient should be in a high-fowler's position, which will maximize the lung capacity. Vital signs, level of pain and an adequate oxygenation must be evaluated.

Nurses are going to remove the tape that secure the orotracheal tube and then deflate the cuff. Usually, removing the tape requires two nurses, in order to prevent premature tube removal.

During extubation, the patient is encouraged to cough while the tube is being removed. Then, the patient will be encouraged to cough again, in order to promote mobilization of secretions.

\section{Post-extubation interventions discussion}

The patient should remain in a high-fowler position and immediately after extubation a supplement oxygen via mask will be provided. Oxygenation levels must be measured, and nurses must encourage patients to take deep breaths to promote ventilation and evaluate signs of respiratory distress, such as increased respiratory 
rate, use of accessory muscles for breathing, cyanosis and increased anxiety.

Regarding to oral hygiene, each quadrant of the mouth must be brushed for 30 seconds, with a small amount of toothpaste applied to toothbrush, twice a day. The tongue must also be scraped from posterior to anterior using a tongue scraper and the patient must swish and spit a mouth rinse. All teeth must be flossed and nurses should apply a lip balm. Oral health is linked to systemic health, disease risk, self-concept, and social interactions, as well as comfort and nutrition and these interventions are essential to prevent ventilator-associated pneumonia, to promote well-being and comfort and to reduce the number of pathogenic organisms (Figure 2).

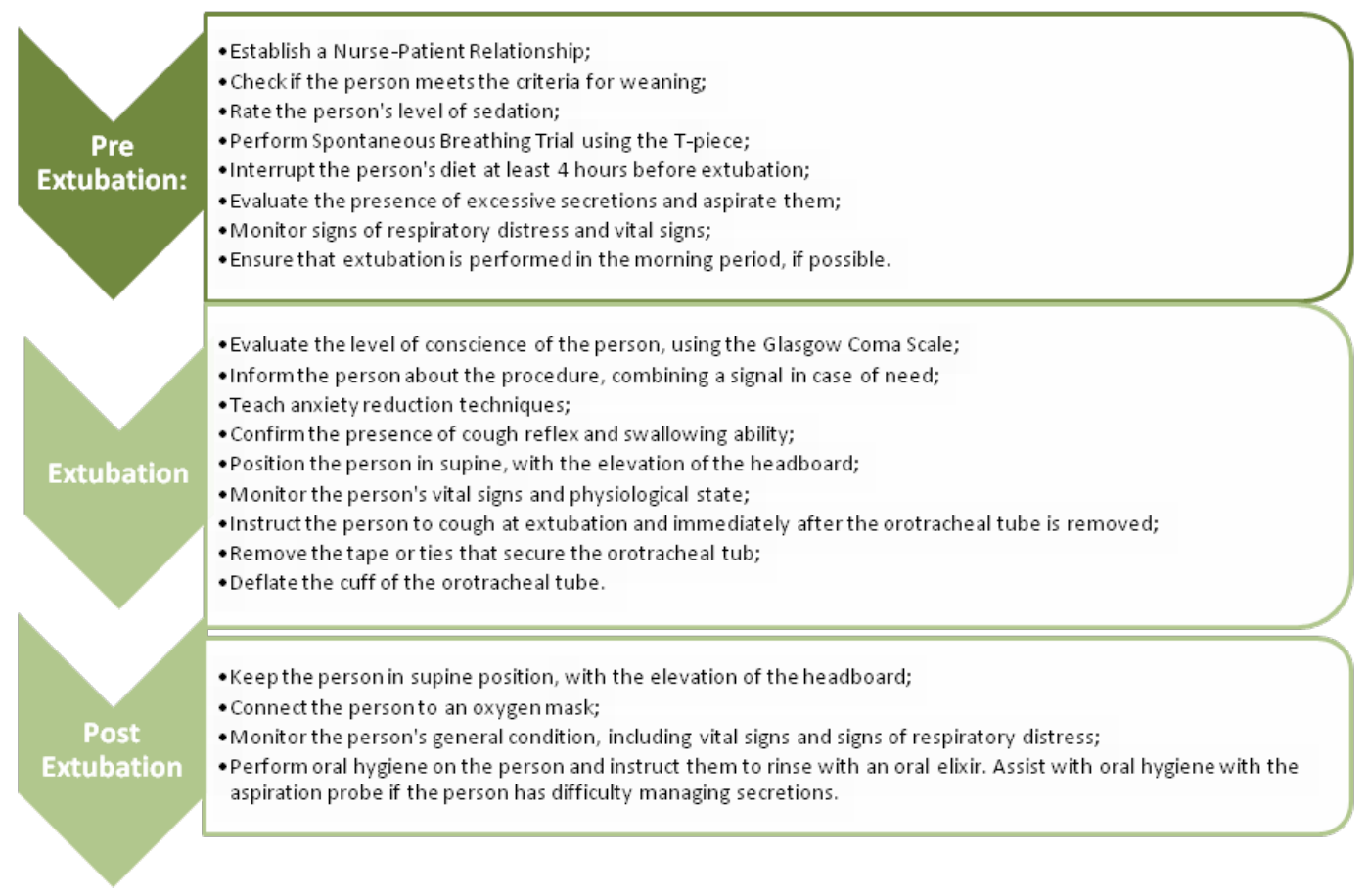

Figure 2 Discussion of the results.

\section{Limitations of the work}

This scoping review only included studies in Portuguese and English language, so studies published in other languages were not analyzed or added according to their contributions. Thus, it is not an exhaustive review of all data sources.

The following scheme presents the nursing interventions succinctly, in order to optimize the extubation process in orotracheally intubated person in intensive care unit, allowing us to answer the research question initially performed.

The existing information about the extubation process, in most of the studies analyzed, was not presented synthetically, which made the process of data extraction harder.

\section{Conclusion}

The complexity of the invasive mechanical ventilation and extubation process requires a differentiated intervention from the nursing team that responds to the needs of each patient.

Nursing research enables health professionals to gain practicerelated knowledge that empower them to make more appropriate and informed decisions.Nursing care is essential for people submitted to invasive mechanical ventilation and it is necessary to adapt the nurse's interventionsand care process.

The role of the nurse towards the person is fundamental as an individual, a member of a familyand in the community in which he is inserted with the objective of responding to the respiratory process and to reduce suffering, improvinglife quality.

It is shown that the vision and the holistic intervention during the extubation process is essential for the improvement of the quality of the ventilatory weaning and consequent extubation. This study allows for gathering the best existing scientific evidence to provide nursing cares of quality and excellence, minimizing the associated risks, by informing the reader in a schematic form, of the nursing care in the various stages of the extubation process.

\section{Acknowledgments}

None.

\section{Conflicts of interest}

There are no conflicts of interest to declare.

\section{References}

1. Simões JA. Intervenções do enfermeiro especialista em enfermagem de reabilitaçãoà pessoa submetida a ventilação mecânica invasiva.(masther thesis) lisbon: lisbon nursing school. 2016.

2. Carvalho CR, Junior CT, Franca SA. Ventilação mecânica: princípios, análise gráfica e modalidades ventilatórias. Brazil: São Paulo. 2007.

3. Mota JS, Zacharias MA. Complicações relacionadas ao tempo de ventilação mecânica invasiva em pacientes com avc internados na uci do hospital universitário são francisco.Portugal: San Francisco University. 2017. 
4. Fogaça LS, Silva GM. Assistência de enfermagem ao paciente sobventilação mecânica controlada. Educational Foundation of Assis. 2017.

5. Jesus, EG, Santo FR. Cuidados de enfermagem ao cliente em uso de ventilação mecânica invasiva. Updated Library. Brazil: Federal University of Bahia. 2017

6. Marcelino P. Manual de ventilação mecânica no adulto - abordagem ao doente crítico. Loures: Lusociencia. 2008.

7. Epstein SK, Ciubotaru RL, Wong JB. Effect of failed extubation on the outcome of mechanical ventilation. Boston: Tufts University School of Medicine. 1997.

8. Melo EM, Teixeira CS, Oliveira RT, et al. Cuidados de enfermagem ao utente sob ventilação mecânica internado em unidade de terapia intensiva. Brazil:Nursing Reference Magazine. 2017;55-63.

9. Craig, JV, Smyth RL. Prática baseada na evidência manual para enfermeiros. Loures:Lusociência. 2004.

10. Ribeiro J. Revisão de investigação e evidência científica.portugal: faculty of psychology and educational sciences of the university of porto. 2014.

11. Khalafi A, Elahi N, Ahmadi F. Continuous care and patients'basic needs duringweaning from mechanical ventilation: A qualitative study. Iran: Faculty of Sciences Doctors: Department of Nursing. 2016.
12. Ward D, Fulbrook P. Nursing strategies for effective weaning of the critically Ill mechanically ventilated patient. Australia. 2016.

13. Oliveira S. Impacto de um protocolo de desmame ventilatório para adultos numa unidade de cuidados intensivos do norte de portugal. (Master Thesis) Trás-os-Montes e Alto Douro University/University of Minho: Vila Real Nursing School. 2017.

14. Caple C, Schub E. T-Piece: using for spontaneous breathing (Weaning) trial. california: glendale: cinahl information systems. 2018

15. Caple C, Heering H. Mechanical ventilation: weaning using pressureadult. california: glendale: cinahl information systems. 2017.

16. Avital O, Schub T. Extubation, Adult: Assisting with. California: Glendale. 2017.

17. Lange M, Badjatia N, Chang W. Implementation of a clinical pathway to reduce rates of postextubation stridor. United States of America: American Association of Critical-Care Nurses. 2018;38(5):34-41.

18. Chu CC, Liu CJ, Yen SM, et al. Factors associated with reintubation within 14 days after ventilator liberation. respiratory care. 2017;62(12):1557-1564

19. Chipps E, Carr M, Kearney RJ, et al. Outcomes of an Oral Care Protocol in Postmechanically Ventilated Patients. World views on EvidenceBased Nursing. 2017;102-111. 